[2] Westerweel PE, Luyten RK, Koomans HA, Derksen RH, Verhaar MC. Premature atherosclerotic cardiovascular disease in systemic lupus erythematosus. Arthritis Rheum. 2007 May;56(5):1384-96.

[3] Shamseer L, Moher D, Clarke M, Ghersi D, Liberati A, Petticrew M, Shekelle P, Stewart LA; PRISMA-P Group. Preferred reporting items for systematic review and meta-analysis protocols (PRISMA-P) 2015: elaboration and explanation. BMJ. 2015 Jan 2;350:g7647.

Disclosure of Interests: None declared

DOI: 10.1136/annrheumdis-2021-eular.2008

\section{AB0082 PERSISTENT LOW COMPLEMENT LEVELS AND INTER FERON GENE UPREGULATION ARE PREDICTIVE FOR DISEASE PROGRESSION IN PATIENTS WITH INCOM- PLETE SYSTEMIC LUPUS ERYTHEMATOSUS}

W. Lambers ${ }^{1}$, J. Westra ${ }^{2}$, S. Arends ${ }^{2}$, B. Doornbos- van der Meer ${ }^{2}$, B. Horvath ${ }^{3}$, H. Bootsma ${ }^{2}$, K. De Leeuw ${ }^{2} .{ }^{1}$ University Medical Centre Groningen, Rheumatology/Clinical Immunology, Groningen, Netherlands; ${ }^{2}$ University Medical Center Groningen, Rheumatology/Clinical Immunology, Groningen, Netherlands; ${ }^{3}$ University Medical Center Groningen, Dermatology, Groningen, Netherlands

Background: A subgroup of lupus patients present with mild symptoms and immunologic features, while they do not meet classification criteria for SLE. This disease state can be referred to as "incomplete systemic lupus erythematosus" (iSLE). Up to $55 \%$ of iSLE patients progress to SLE. Furthermore, previous research has shown that iSLE might overlap with early primary Sjögren's disease (pSS).(1) Unfortunately, there are no predictive markers available for progression to classifiable disease. Type-l interferon (IFN) plays an important role in disease initiation of both SLE and pSS. $(2,3)$ Myxovirus-resistance protein A $(M \times A)$ is a GTP-ase that has previously be demonstrated to correlate strongly with IFN-type I expression. Furthermore, interferon-inducible chemokines IFN- $\gamma$ induced protein 10 (IP-10), and B-cell activating factor (BAFF), that are both inducible by IFN, are of interest, because it is demonstrated that these proteins are increased prior to the diagnosis of SLE.(4)

Objectives: To find predictive markers that identify patients with incomplete systemic lupus erythematosus (iSLE) who are at the highest risk to progress to classifiable systemic lupus erythematosus (SLE) or primary Sjögren's syndrome (pSS).

Methods: Patients with iSLE (ANA $\geq 1: 80, \geq 1$ clinical SLICC criterion, but not fulfilling the criteria, and disease manifestation $<5$ years) were included in a longitudinal observational study. Every half year, clinical status was evaluated and regular immunological serologic assessment was performed. Annually, interferon (IFN)-gene expression was determined by RT-PCR in whole blood using 14 genes. These genes represented 3 IFN-related modules. Some genes were mainly inducible by IFN-type I, others by IFN-type II. Furthermore, IFN-related mediators Myxovirus resistance protein A (MxA), interferon-gamma-induced protein 10 (IP-10) and B-cell activating factor (BAFF) were measured.

Results: Of 38 included iSLE patients, 6 had developed SLE and 1 develop pSS $(18 \%)$ after median follow up of 36 months. The 7 patients who developed SLE/pSS were all women, and were younger at baseline than those who remained having iSLE (median 26 years, IQR $20-29$ vs. median 42 years, IQR $30-56, p=0.0009)$. Over time, these patients had significantly lower complement $3(p<0.0001)$ and complement 4 levels $(p=0.005)$, higher IFN-gene expression $(p=0.007)$, and lower neutrophil counts $(p=0.033)$ (see Figure 1.). No difference was found between IFN-type I and IFN-type II inducible genes. Levels of MxA, IP-10 and BAFF did not differ between patients who remained iSLE and who progressed to SLE/pSS.

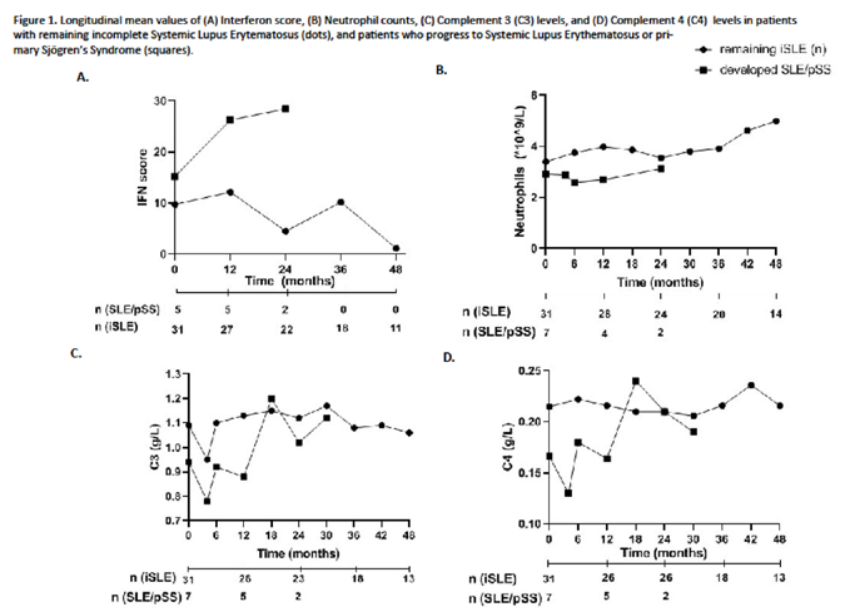

Figure 1.
Conclusion: Gender, age at diagnosis, persistent low complement levels, and high IFN-gene expression can help to identify iSLE patients at the highest risk of progressing to classifiable disease.

\section{REFERENCES:}

[1] Md Yusof MY, et al. Prediction of autoimmune connective tissue disease in an at-risk cohort: Prognostic value of a novel two-score system for interferon status. Ann Rheum Dis. 2018;1-8.

[2] Yao Y, et al. Type I interferons in Sjögren's syndrome. Autoimmun Rev. 2013;12(5):558-66.

[3] Crow MK. Type I Interferon in the Pathogenesis of Lupus. J Immunol [Internet]. 2014;192(12):5459-68.

[4] Lu R, et al. Dysregulation of innate and adaptive serum mediators precedes systemic lupus erythematosus classification and improves prognostic accuracy of autoantibodies. J Autoimmun. 2016;74:182-93.

Disclosure of Interests: None declared

DOI: 10.1136/annrheumdis-2021-eular.2306

\section{AB0083 SIGNALING OF PSGL-1 IN HUMAN NEUTROPHILS MIGHT CONTROL NET GENERATION AND APOPTOSIS AND MIGHT BE ALTERED IN SLE PATIENTS}

A. Muñoz-Callejas ${ }^{1}$, E. González Sánchez ${ }^{1}$, E. F. Vicente-Rabaneda ${ }^{2}$, J. Garcia Perez $^{3}$, S. Castañeda ${ }^{2}$, A. Urzainquii . 'Instituto de Investigación SanitariaHospital de la Princesa, Inmunologia, Madrid, Spain; ${ }^{2}$ Hospital de la Princesa, Reumatologia, Madrid, Spain; ${ }^{3}$ Hospital de la Princesa, Neumologia, Madrid, Spain

Background: Neutrophil extracellular traps (NETs) are extracellular chromatin fibers decorated with neutrophil antimicrobial proteins and histones, which are formed by neutrophils to trap invading pathogens and facilitate their removal. Excessive presence of NETs has been reported in autoimmune diseases like Systemic Lupus Erythematosus (SLE) and has been related with disease pathogenesis [1]. Our previous work showed that P-selectin KO mice develop an autoimmune syndrome similar to human lupus and that patients with cutaneous lupus have reduced expression of P-selectin in skin vessels [2]. Although it has been reported that $P$-selectin might induce NET generation in mouse neutrophils [3] there are no studies performed with human neutrophils.

Objectives: 1)To analyze the contribution of PSGL-1/P-Selectin interaction to control the generation of NETs by human neutrophils from healthy donors and patients with SLE.

2)To study the implication of PSGL-1/P-Selectin interaction in the control of other types of neutrophil death.

Methods: Human neutrophils were isolated from healthy donors and patients with SLE. After incubation in vitro with BSA or P-Selectin in rolling-like conditions (60 rpm shaking at $37^{\circ} \mathrm{C}$ ) for $10 \mathrm{~min}$, neutrophils were left to adhere for 1 hour. Then, NETs were labeled with Sytox Green and quantified by fluorimetry and by flow cytometry (FACSCanto II, BD Biosciences). For quantification of necrosis and apoptosis, cells were labeled with propidium iodide and annexing $\mathrm{V}$ and analyzed by flow cytometry. Data were analyzed with two-sided Student, twosided Mann-Whitney U or two-sided Wilcoxon signed rank test using GraphPad Prism software (Version 8.0.1, La Jolla, CA). Results were considered significant at $\mathrm{P}<.05$.

Results: Our results show that rolling on BSA, compared to adhesion, induces NET generation and apoptosis of neutrophils isolated from blood of healthy donors. Rolling on P-selectin reduces apoptosis of neutrophils and increase the percentage of NET events, although reducing NET intensity. Comparing to healthy donors, neutrophils from active SLE patients generate NETs with higher intensity in adhesion and do not respond to P-Selectin.

Conclusion: The interaction of PSGL-1 with P-Selectin preserves neutrophil death from apoptosis and controls NET generation. Our results suggest that this control might be altered in patients with active SLE.

REFERENCES:

[1] Fousert, E., Toes, R., \& Desai, J. (2020). Neutrophil extracellular traps (NETs) take the central stage in driving autoimmune responses. Cells, 9(4), 915.

[2] González-Tajuelo, R., Silván, J., Pérez-Frías, A., De La Fuente-fernández, M., Tejedor, R., Espartero-Santos, M. \& Urzainqui, A. (2017). P-Selectin preserves immune tolerance in mice and is reduced in human cutaneous lupus. Scientific reports, 7(1), 1-12.

[3] Etulain, J., Martinod, K., Wong, S. L., Cifuni, S. M., Schattner, M., \& Wagner, D. D. (2015). P-selectin promotes neutrophil extracellular trap formation in mice. Blood, 126(2), 242-246.

Disclosure of Interests: None declared

DOI: 10.1136/annrheumdis-2021-eular.2508 